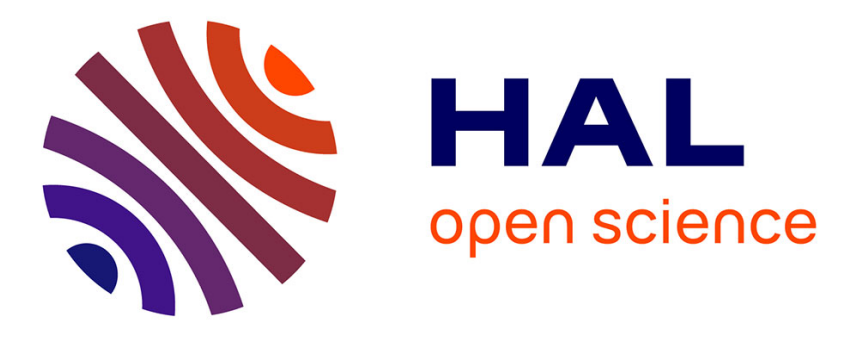

\title{
Systematic study of the spin stiffness dependence on phosphorus alloying in the ferromagnetic semiconductor (Ga,Mn)As
}

S Shihab, H Riahi, L. Thevenard, H J von Bardeleben, Aristide Lemaître, C. Gourdon

\section{To cite this version:}

S Shihab, H Riahi, L. Thevenard, H J von Bardeleben, Aristide Lemaître, et al.. Systematic study of the spin stiffness dependence on phosphorus alloying in the ferromagnetic semiconductor (Ga,Mn)As. Applied Physics Letters, 2015, 106 (14), pp.142408. 10.1063/1.4917423 . hal-01416609

\author{
HAL Id: hal-01416609 \\ https://hal.science/hal-01416609
}

Submitted on 14 Dec 2016

HAL is a multi-disciplinary open access archive for the deposit and dissemination of scientific research documents, whether they are published or not. The documents may come from teaching and research institutions in France or abroad, or from public or private research centers.
L'archive ouverte pluridisciplinaire HAL, est destinée au dépôt et à la diffusion de documents scientifiques de niveau recherche, publiés ou non, émanant des établissements d'enseignement et de recherche français ou étrangers, des laboratoires publics ou privés. 


\section{AIP Appled phy \\ Letters}

\section{Systematic study of the spin stiffness dependence on phosphorus alloying in the ferromagnetic semiconductor (Ga,Mn)As}

S. Shihab, H. Riahi, L. Thevenard, H. J. von Bardeleben, A. Lemaître, and C. Gourdon

Citation: Applied Physics Letters 106, 142408 (2015); doi: 10.1063/1.4917423

View online: http://dx.doi.org/10.1063/1.4917423

View Table of Contents: http://scitation.aip.org/content/aip/journal/apl/106/14?ver=pdfcov

Published by the AIP Publishing

\section{Articles you may be interested in}

Ferromagnetic $(\mathrm{Ga}, \mathrm{Mn})$ As nanowires grown by $\mathrm{Mn}$-assisted molecular beam epitaxy

J. Appl. Phys. 113, 144303 (2013); 10.1063/1.4799624

Coherent manipulation of magnetization precession in ferromagnetic semiconductor $(\mathrm{Ga}, \mathrm{Mn})$ As with successive optical pumping

Appl. Phys. Lett. 93, 202506 (2008); 10.1063/1.3030988

Effect of Be doping on the properties of GaMnAs ferromagnetic semiconductors

J. Appl. Phys. 93, 8307 (2003); 10.1063/1.1556272

Ferromagnetic semiconductor heterostructures based on (GaMn)As

J. Vac. Sci. Technol. A 18, 1247 (2000); 10.1116/1.582334

Spin-dependent tunneling and properties of ferromagnetic (Ga,Mn)As (invited)

J. Appl. Phys. 85, 4277 (1999); 10.1063/1.370343

You don't

still use this

cell phone

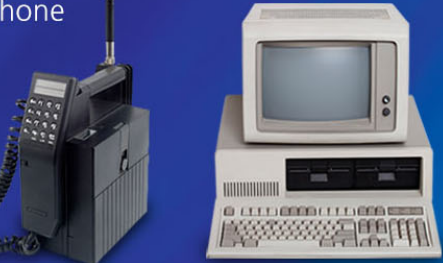

Why are you still using an AFM designed in the 80 's?
It is time to upgrade your AFM

Minimum $\$ 20,000$ trade-in discount for purchases before August 31st

Asylum Research is today's technology leader in AFM 


\title{
Systematic study of the spin stiffness dependence on phosphorus alloying in the ferromagnetic semiconductor $(\mathrm{Ga}, \mathrm{Mn}) \mathrm{As}$
}

\author{
S. Shihab, ${ }^{1,2}$ H. Riahi, ${ }^{3}$ L. Thevenard,${ }^{1,2}$ H. J. von Bardeleben, ${ }^{1,2}$ A. Lemaître, ${ }^{4}$ \\ and C. Gourdon ${ }^{1,2}$ \\ ${ }^{1}$ CNRS, UMR7588, Institut des Nanosciences de Paris, 4 place Jussieu, 75005 Paris, France \\ ${ }^{2}$ Sorbonne Universités, UPMC Université Paris 06, UMR7588, 4 place Jussieu, 75005 Paris, France \\ ${ }^{3}$ Laboratoire Matériaux Molécules et Applications, IPEST, Université de Carthage, B.P. 51, 2075 La Marsa, \\ Tunisia \\ ${ }^{4}$ Laboratoire de Photonique et Nanostructures, CNRS, UPR 20, Route de Nozay, 91460 Marcoussis, France
}

(Received 9 February 2015; accepted 1 April 2015; published online 10 April 2015)

\begin{abstract}
We study the dependence of the spin stiffness constant on the phosphorus concentration in the ferromagnetic semiconductor $(\mathrm{Ga}, \mathrm{Mn})(\mathrm{As}, \mathrm{P})$ with the aim of determining whether alloying with phosphorus is detrimental, neutral, or advantageous to the spin stiffness. Time-resolved magnetooptical experiments are carried out in thin epilayers. Laser pulses excite two perpendicular standing spin wave modes, which are exchange related. We show that the first mode is spatially uniform across the layer corresponding to a $k \approx 0$ wavevector. From the two frequencies and k-vector spacings we obtain the spin stiffness constant for different phosphorus concentrations using weak surface pinning conditions. The mode assessment is checked by comparison to the spin stiffness obtained from domain pattern analysis for samples with out-of-plane magnetization. The spin stiffness is found to exhibit little variation with phosphorus concentration in contradiction with ab-initio predictions. (C) 2015 AIP Publishing LLC. [http://dx.doi.org/10.1063/1.4917423]
\end{abstract}

Building complex heterostructures, such as tunnel magnetic junctions, from the same host material is a challenge in order to reduce detrimental interface effects between different parts of a spintronic device. In this framework, diluted magnetic semiconductors (DMS) are a class of materials able to address this challenge. ${ }^{1}$ More fundamentally, DMS and more specifically the III-V based (Ga,Mn)As have become in the past decade a benchmark material in order to achieve predictable tuning of magnetic properties. Levers such as the temperature, ${ }^{2}$ the carrier concentration ${ }^{3,4}$ but also the strain applied on the magnetic layer ${ }^{5,6}$ or alloying with phosphorus ${ }^{7,8}$ have been used in order to change the micromagnetic properties, e.g., the Curie temperature $T_{C}$, the saturation magnetization $M_{s}$, and the magnetic easy axis.

Among these properties, the spin stiffness $D$ is perhaps the most difficult to tune, despite theoretical guidelines. ${ }^{9}$ An increase of the spin stiffness keeping constant magnitude of the magnetization $M_{s}$ would mean larger exchange constant $A$ $\left(A=D M_{s} / 2\right)$ and therefore larger domain wall width and domain wall velocity, this tunability remaining out of reach for metals. It is expected that alloying with phosphorus should increase the spin stiffness owing to an increase of Mn-hole exchange integral $J_{p d}$, the stronger hybridization of the p-d wave functions arising mainly from the smaller lattice constant of GaMnP. ${ }^{10}$ The effect on $T_{C}$ remains theoretically unclear. Whereas an overall increase is predicted from $(\mathrm{Ga}, \mathrm{Mn}) \mathrm{As}$ to $(\mathrm{Ga}, \mathrm{Mn}) \mathrm{P},{ }^{10,11}$ a decrease might occur for $\mathrm{P}$ concentration between zero and $25 \%{ }^{11}$ Furthermore, it was shown experimentally that $\left(\mathrm{Ga}_{1-x}, \mathrm{Mn}_{x}\right)\left(\mathrm{As}_{1-y}, \mathrm{P}_{y}\right)$ suffers a metal-to-insulator transition with increasing phosphorus concentration with a drop of its $T_{C}{ }^{12,13}$ A modest increase of $D$ between $y=0$ and $y>6 \%$ can be inferred from results on the exchange constant $A$ obtained for $(\mathrm{Ga}, \mathrm{Mn})(\mathrm{As}, \mathrm{P})$ samples with out-of-plane (OP) easy axis using domain pattern analysis. ${ }^{14,15}$ However, recent results using an optical technique suggest a decrease of $D$ with $\mathrm{P}$ alloying but with only one $(\mathrm{Ga}, \mathrm{Mn})(\mathrm{As}, \mathrm{P})$ sample studied. ${ }^{16}$ Unfortunately domain pattern analysis cannot be used to determine the spin stiffness for in-plane (IP) magnetized samples. To assess this value at low $\mathrm{P}$ concentration and in-plane easy axis, techniques based on the excitation of exchange related perpendicular standing spin waves (PSSW) can instead be used. The spin stiffness constant can be extracted from the frequency spacing of excited modes. One technique is the standard ferromagnetic resonance (FMR), ${ }^{4,17}$ another is the time-resolved magnetooptical Kerr effect experiment (TRMOKE) ${ }^{6,16,18,19}$ recently pointed out as an optical analog of FMR for DMS. ${ }^{19}$ However, experimentally some modes undetectable by FMR are observed by TRMOKE as will be shown further on.

Here, we report on the determination of the spin stiffness constant by TRMOKE for samples with several $\mathrm{P}$ and Mn concentrations. In order to assess the mode k-vector we use the results previously obtained from domain pattern analysis for out-of-plane magnetized samples. ${ }^{15}$ We obtain the variation of $D$ with phosphorus concentration up to $9 \%$.

Samples used for this study are epilayers of $\left(\mathrm{Ga}_{1-x}, \mathrm{Mn}_{x}\right)$ $\left(\mathrm{As}_{1-y}, \mathrm{P}_{y}\right)$ grown on a (001) GaAs substrate and annealed $1 \mathrm{~h}$ at $250^{\circ} \mathrm{C}$. The thickness obtained from X-ray measurements is in the range of $43-50 \mathrm{~nm}$. Samples from two sets with effective Mn concentration $x_{\text {eff }}$ around $3.5 \%$ and $5 \%$ were studied. $x_{\text {eff }}$ was determined from the saturation magnetization $\mathbf{M}_{s}$ measured by SQUID magnetometry. All samples were characterized beforehand by FMR. ${ }^{8}$ Typical FMR spectra are displayed in the inset of Fig. 1, where only one PSSW mode is observed, as for most samples. Actually, for a layer with homogeneous magnetization and magnetic anisotropy, only spin wave modes with a non-zero integral over the layer thickness of the time-dependent magnetization component should be 


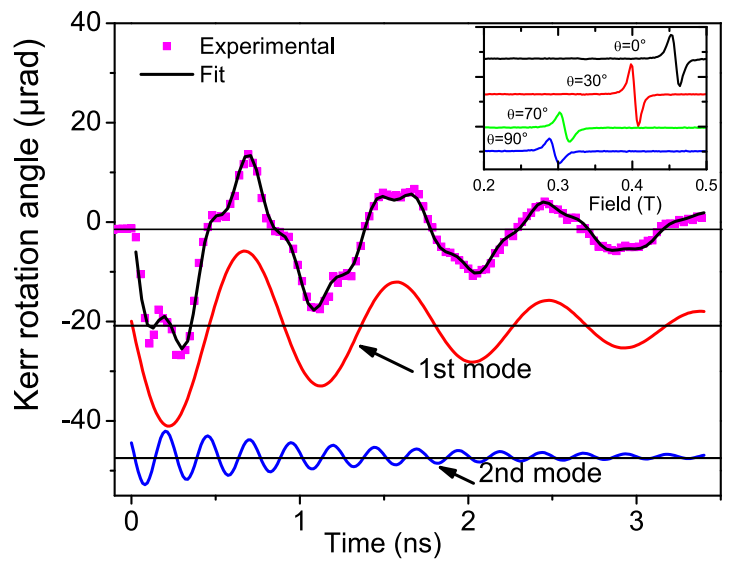

FIG. 1. Typical dynamical signal obtained in TRMOKE experiments. The top curve displays the experimental signal (squares) and the associated fit (solid line) for $(\mathrm{Ga}, \mathrm{Mn})(\mathrm{As}, \mathrm{P})$ with $x_{\text {eff }}=3.6 \%$ and $y=3.4 \%$ at $T=12 \mathrm{~K}$. The two curves below represent the oscillatory components of the experimental signal obtained from the fit of the signal, shifted for clarity. Inset: FMR spectra at $T=4 \mathrm{~K}$ for different angles of an out-of-plane field ( $\theta$ with respect to the [001] direction).

excited by a uniform radio-frequency field. ${ }^{20}$ Their detection would however depend on the signal level with respect to noise. Observation of only the fundamental mode allows the determination of the magnetic anisotropy constants but precludes any estimation of the spin stiffness constant. TRMOKE experiments are carried out on in-plane magnetized samples with zero external magnetic field after a $60 \mathrm{mT}$ initial preparation step. For out-of-plane samples an in-plane field is applied to pull the magnetization into the layer plane. The laser source is a Ti:Sa laser with pulse width $\approx 200 \mathrm{fs}$ at wavelength $\lambda=703 \mathrm{~nm}$. The sample is glued on the cold finger of a liquid He flow cryostat. To limit thermal effects, experiments are performed at low pump and probe fluence $\left(\mathrm{F}_{\text {pump }}=1.1 \mu \mathrm{J}\right.$ $\mathrm{cm}^{-2}, \mathrm{~F}_{\text {probe }}=0.4 \mu \mathrm{J} \mathrm{cm}^{-2}$ ), with a circularly or linearly pump beam, and a probe beam linearly polarized along the magnetization direction. At low temperature, a pump induced stationary increase of temperature of $0.5 \mathrm{~K}$ is estimated. The magnetization dynamics is monitored through the Kerr rotation of the polarization detected by a balanced optical bridge.

A typical dynamical signal is shown in Fig. 1. After excitation by the pump beam at $\mathrm{t}=0$, which generates a transient change of the anisotropy constants and therefore of the effective magnetic field, the magnetization is launched into precession and relaxes toward its equilibrium position in a few ns. The dynamical signal exhibits damped oscillations with two frequencies, representing optically generated spin wave modes (in some samples only one frequency is detected). For most of the samples, there is no difference between linearly and circularly polarized pump, except for two samples without $\mathrm{P}$ where the second spin-wave frequency was only observed in the helicity dependent signal. The TRMOKE signal is fitted by the relation

$$
S(t)=\sum_{i} A_{i} e^{-\alpha_{i} 2 \pi f_{i} t} \sin \left(2 \pi f_{i} t+\phi_{i}\right),
$$

where $\mathrm{f}_{i}$ is the frequency, $\phi_{i}$ the phase, $\mathrm{A}_{i}$ the amplitude, and $\alpha_{i}$ the effective (inhomogeneous) damping of the i-th excited mode. The signal in Fig. 1 is obtained in a $y=3.4 \%$ sample at $12 \mathrm{~K}$. Parameters from the fit are $f_{1}=1.11 \pm 0.02 \mathrm{GHz}$, $f_{2}=4.03 \pm 0.05 \mathrm{GHz}$, and $\alpha_{1,2}=0.08$, and the amplitude ratio between the two modes is 0.3 .

To extract $D$ from the observed frequencies, the magnetization dynamics is modeled in a standard way, starting from the Landau-Lifshitz-Gilbert equation with the exchange field and without damping. ${ }^{6,18}$ For in-plane easy-axis, the equations for small precession angle of the magnetization vector read

$$
\begin{gathered}
\dot{\delta \theta}=\gamma\left[-F_{\phi \phi} \delta \phi / M_{s}+D \frac{\partial^{2} \delta \phi}{\partial z^{2}}\right] \\
\dot{\delta \phi}=\gamma\left[F_{\theta \theta} \delta \theta / M_{s}-D \frac{\partial^{2} \delta \theta}{\partial z^{2}}\right],
\end{gathered}
$$

where the $z$-axis $(||[001])$ is perpendicular to the layer plane, and $\theta$ and $\phi$ are the polar and the azimuthal angles, respectively. $F_{i j}=\left.\frac{\partial^{2} F}{\partial i \partial j}\right|_{\phi=\phi_{0}}$ are the second derivatives of the magnetic energy with respect to the spherical coordinates using FMR convention ${ }^{22}$ for the anisotropy constants $K_{i}$

$$
\begin{aligned}
F_{\theta \theta}= & -2 K_{2 \perp}+K_{4 \|}\left(3+\cos 4 \phi_{0}\right) / 2 \\
& +2 K_{2 \|}\left(1-\sin 2 \phi_{0}\right)+\mu_{0} M_{s}^{2} \\
F_{\phi \phi}= & 2\left(K_{4 \|} \cos 4 \phi_{0}-K_{2 \|} \cos 2 \phi_{0}\right)
\end{aligned}
$$

with $\phi_{0}$ the equilibrium angle with respect to [100] given by $\sin 2 \phi_{0}=-K_{2 \|} / K_{4 \|}$ if $\left|K_{2 \|} / K_{4 \|}\right|<1$ and $\phi_{0}=\pi / 4$ otherwise. For an out-of-plane easy axis and an in-plane applied magnetic field, the above equations are modified to include the field. Calculations of the time dependent part give the magnon dispersion relation

$$
f(k)=\frac{\gamma}{2 \pi} \sqrt{F_{\theta \theta} F_{\phi \phi} / M_{s}^{2} \pm\left(F_{\theta \theta}+F_{\phi \phi}\right) D k^{2} / M_{s}+D^{2} k^{4}},
$$

where $\gamma$ is the gyromagnetic ratio. The plus sign refers to a bulk mode $(\cos k z, \sin k z)$ and the minus one to a surface mode $(\cosh k z, \sinh k z)$. Note that by setting $D=0$, one recovers the Smit-Beljers formula used for FMR. ${ }^{23}$ The dispersion curve for a sample with $y=4.3 \%$ at $T=12 \mathrm{~K}$ and $H=0$ is displayed in Fig. 2(a). The $k=0$ frequency represents the spatially uniform mode and is related only to the anisotropy constants, while the curvature of the bulk mode dispersion curve depends on $D$.

The spatial dependence of $\delta \theta$ and $\delta \phi$ is calculated by finding the allowed confined surface-bulk hybridized spin wave wavevectors for a sample thickness $L$. Following Refs. 6 and 18, Rado-Weertman symmetric general boundary conditions for an in-plane magnetization are used ${ }^{24}$

$$
\left.\frac{\partial \delta \phi}{\partial z}\right|_{ \pm L / 2}=\left.0 \frac{\partial \delta \theta}{\partial z}\right|_{ \pm L / 2}=\mp \frac{2 K_{s}}{D M_{s}} \delta \theta,
$$

where $F_{s}=K_{s} \cos ^{2} \theta$ is the surface anisotropy energy acting as a pinning term hindering the surface spin precession. The natural freedom condition $\left(K_{s}=0\right)$ gives a $k$-quantification in $n \pi / L$, where even (odd) $n$ correspond to even (odd) spin 

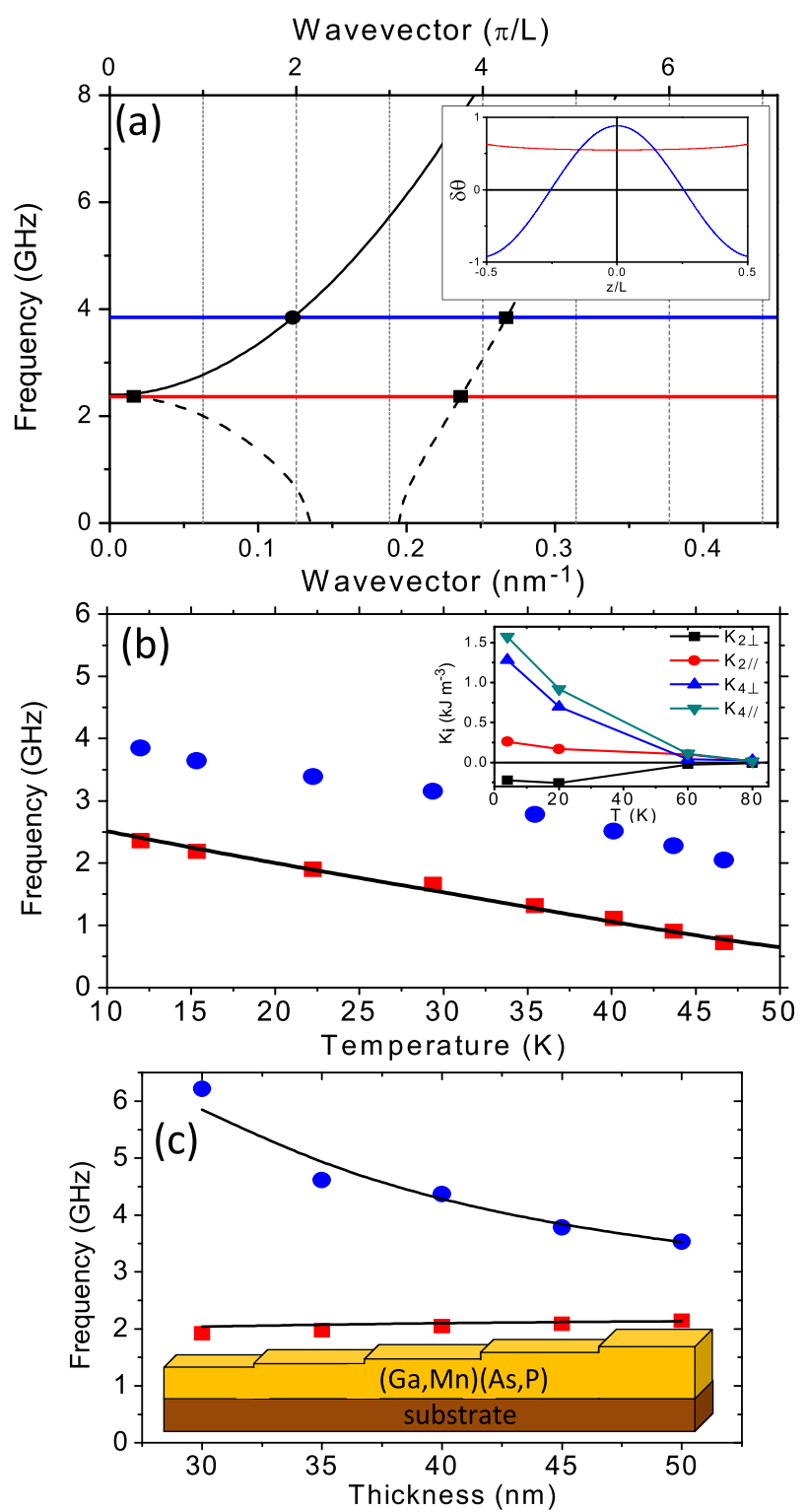

FIG. 2. Sample with $y=4.3 \%$. (a) Magnon dispersion relation showing the bulk modes (solid black line) and the surface modes (dashed black line) $(L=50 \mathrm{~nm})$. Horizontal lines represent the experimental frequencies ( $T=12 \mathrm{~K}$ ). Squares (circles) represent the wavevectors of the bulk (surface) components of the spin waves found from the boundary conditions (Eq. (6)). Inset: Mode profile (out-of-plane component $\delta \theta$ ) across the layer thickness for the first and second excited modes. (b) Frequencies of spin wave modes from TRMOKE (symbols) as a function of temperature, compared with the $k=0$ frequency (solid line) calculated from FMR anisotropy constants (inset). (c) Layer thickness dependence of the frequencies of the two spin wave modes observed in TRMOKE experiments $(T=12 \mathrm{~K})$. The solid lines are the frequencies calculated from Eqs. (5) and (6) using $D=3.4 \mathrm{~T} \mathrm{~nm}^{2}$, and the anisotropy constants from FMR (inset of (b)). A sketch of the staircase-like sample after etching is shown below the curves.

wave modes. Given the experimental precession frequencies, the spin stiffness and the mode profiles are obtained through an iterative adjustment of $D$ and $K_{s}$ (Fig. 2(a)). $D$ determines the frequency spacing between the two modes, while the pinning constant $K_{s}$ shifts their frequencies.

In order to run this model, a preliminary step is to check the spatial profile of the lowest energy mode along the same lines as in Ref. 19. To that end, a wet etching procedure was carried out on a $y=4.3 \%$ sample in order to obtain a staircase pattern (Fig. 2(c)). Successive oxidation and oxide removal sequences lead to a thickness difference of $5 \mathrm{~nm}$ between each step. The experimental results for this sample (Fig. 2(c)) show that the first mode frequency does not change with decreasing thickness while the second one varies with the thickness as expected from Eqs. (5) and (6). This shows first that the sample magnetic properties have a good in-depth and lateral uniformity and, second, that we are observing two exchange related PSSWs, where the first mode is a quasi-uniform mode as found from the mode profile calculation (inset of Fig. 2(a)). Indeed, we find a very small surface anisotropy constant $K_{s}=-1.3 \mu \mathrm{J} \mathrm{m}^{-2}$, which divided by the layer thickness gives an equivalent bulk anisotropy constant 10 times smaller than the smallest bulk one (inset of Fig. 2(b)).

To compare FMR and optical experiments, we plot the frequencies obtained from TRMOKE and the $k=0$ frequency calculated from the FMR anisotropy coefficients as a function of temperature. The result displayed in Fig. 2(b) shows an excellent agreement between these two experiments for the first mode frequency. Such a comparison was shown for $\mathrm{Nickel}^{21}$ and for $(\mathrm{Ga}, \mathrm{Mn}) \mathrm{As}$ at one temperature only. ${ }^{18}$ Here, a very good agreement on a large temperature range is demonstrated for DMS.

Because two PSSWs are generated, one can obtain $D$ from the frequency spacing. However, the correct value of $D$ depends on the identification of the mode $k$-number (and thus symmetry) for the second excited spin wave. In a TRMOKE experiment, one is not able to discriminate between an odd or even mode. However, according to the arguments developed in Ref. 18, only modes with a non-zero integral of $\delta \theta$ and $\delta \phi$ would be detected. In order to check this point and determine the $k$-number, we compared the $D$ value obtained from stripe domain analysis ${ }^{15}$ for a $y=8.8 \%$ sample (perpendicular easy axis) at $\mathrm{T}=20 \mathrm{~K}, D=11.8 \pm 1.3$ $\mathrm{T} \mathrm{nm}^{2}$, with the $D$ values obtained from TRMOKE (under in-plane field) with the assumption of an even mode, $D=$ $5.4 \pm 0.7 \mathrm{~T} \mathrm{~nm}^{2}$, or an odd mode, $D=21.6 \pm 2.8 \mathrm{~T} \mathrm{~nm}^{2}$. Results from these two experiments are in better agreement with the assumption of an even second mode. The larger $D$ from domain pattern might arise from the assumption of Bloch domain walls whereas they are actually twisted BlochNéel walls. ${ }^{25}$ Moreover, we find that the values obtained from TRMOKE and FMR are in good agreement for this sample, which shows two modes with both techniques (Fig. 3 ), assuming a second even mode also for FMR. This points to a good homogeneity of the sample since TRMOKE is a local probe (a few $\mu \mathrm{m}^{2}$ ) whereas FMR probes the whole layer. Three GaMnAs samples (without phosphorus) have been studied. The $D$ values obtained for two of them from TRMOKE (Fig. 3), and for the third one (on a GaInAs buffer, with perpendicular easy axis) from the width of individual stripe domains close to saturation ${ }^{14}$ are all very close: $4.75 \pm 0.5,4.8 \pm 0.5$, and $5.2 \pm 0.7 \mathrm{~T} \mathrm{~nm}^{2}$, respectively, which again is in favor of an even second PSSW mode in TRMOKE. Let us note that the spin stiffness values for GaMnAs reported in the literature are quite dispersed, some being comparable to ours, ${ }^{17,18}$ others, especially for thicker but likely inhomogeneous samples, being at least 3 times larger, $^{4,18}$ or 4 times larger by TRMOKE but under the 


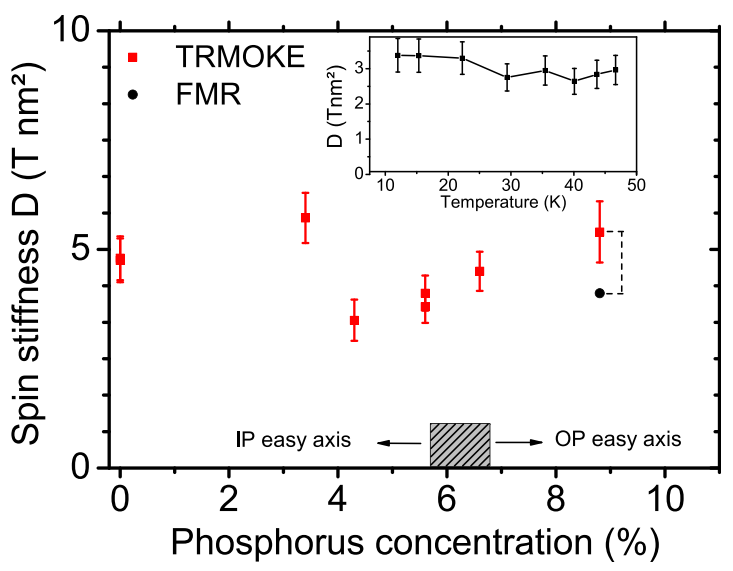

FIG. 3. Dependence of the spin stiffness on the phosphorus concentration. Squares: from TRMOKE (at $T=12 \mathrm{~K}$ ), circles: from FMR. The shaded zone indicates the frontier between samples with IP and OP easy magnetization axis. Data obtained from the same sample by TRMOKE and FMR are linked by a dashed line. The errors bars are calculated from the uncertainty on the epilayer thickness $L$ determined from X-ray measurements (e.g., $L=43-49 \mathrm{~nm}$ for the $\mathrm{y}=8.8 \%$ sample). The inset shows the temperature dependence of $D$ for the sample with $y=4.3 \%$ for which $T_{C}=85 \mathrm{~K}$.

assumption of a second odd mode, ${ }^{16}$ which would give a value close to ours assuming an even second mode.

From TRMOKE results, we obtain $D$ for $(\mathrm{Ga}, \mathrm{Mn})(\mathrm{As}, \mathrm{P})$ samples with $y$ up to $\approx 9 \%$ (Fig. 3). It is worth noticing that for two samples with the same phosphorus concentration $(5.6 \%)$ but different effective Mn concentrations $(3.5 \%$ and $5 \%$ ), we obtain the same spin stiffness. Indeed, by considering the spin stiffness $D=2 A / M_{S}$ and not only $A$ one takes into account the effect of the effective Mn concentration, which ranges here from $3.5 \%$ to $5.2 \%$. Similarly one eliminates the temperature dependence of $A$ and $M_{s}$, which are actually close, ${ }^{15}$ and determines an almost temperature independent quantity (inset of Fig. 3). As can be seen in Fig. 3, D hardly varies with the phosphorus concentration, i.e., with the lattice cell volume, which decreases with $y$. Let us note that while the value of $D$ depends on the mode identification, the trend with phosphorus alloying does not, provided one observes the same pair of modes for all samples. In simple models ${ }^{1} D$ is expected to vary as $p^{1 / 3} J_{p d}^{2}$, where $p$ is the carrier concentration. Our results can mean that $J_{p d}$ does not increase with $y$. The increase of $J_{p d}$ was predicted considering relaxed layers. ${ }^{10}$ However, for pseudomorphic $(\mathrm{Ga}, \mathrm{Mn})(\mathrm{As}, \mathrm{P})$ layers, the lattice cell volume decrease is $40 \%$ smaller, which may diminish the expected variation of $J_{p d}$. Alternatively, a small increase of $J_{p d}$ could be counterbalanced by a decrease of the carrier concentration as suggested in Ref. 13.

As a conclusion, we have carried out time-resolved magneto-optical experiments for $(\mathrm{Ga}, \mathrm{Mn})(\mathrm{As}, \mathrm{P})$ samples. Together with previous results obtained for out-of-plane easy axis samples, these results allow a determination of the spin stiffness constant for phosphorus concentration ranging continuously from zero up to $9 \%$. The spin stiffness is found to vary very weakly with the phosphorus concentration. Incorporation of phosphorus is therefore neither detrimental nor advantageous to increase the spin stiffness.

We are grateful to H. Hurdequint from LPS-Orsay for fruitful discussions, to C. Testelin (INSP) for useful advices, and to M. Bernard, F. Breton and S. Majrab (INSP) for technical assistance. This work has been supported by UPMC (Emergence 2012), by Region Ile-de-France (DIM Nano-K MURAS2012), by Agence Nationale de la Recherche (Nos. ANR 2010-BLANC-0424-02 and ANR13JS04-0001-01), and by the French RENATECH network.

${ }^{1}$ T. Dietl and H. Ohno, Rev. Mod. Phys. 86, 187 (2014).

${ }^{2}$ M. Sawicki, F. Matsukura, A. Idziaszek, T. Dietl, G. Schott, C. Ruester, C. Gould, G. Karczewski, G. Schmidt, and L. Molenkamp, Phys. Rev. B 70, 245325 (2004).

${ }^{3}$ L. Thevenard, L. Largeau, O. Mauguin, and A. Lemaître, Phys. Rev. B 75, 195218 (2007).

${ }^{4}$ C. Bihler, W. Schoch, W. Limmer, S. T. B. Goennenwein, and M. S. Brandt, Phys. Rev. B 79, 45205 (2009).

${ }^{5}$ A. W. Rushforth, E. De Ranieri, J. Zemen, J. Wunderlich, K. W. Edmonds, C. S. King, E. Ahmad, R. P. Campion, C. T. Foxon, B. L. Gallagher, K. Výborný, J. Kučera, and T. Jungwirth, Phys. Rev. B 78, 085314 (2008).

${ }^{6}$ M. Bombeck, A. S. Salasyuk, B. A. Glavin, A. V. Scherbakov, C. Brüggemann, D. R. Yakovlev, V. F. Sapega, X. Liu, J. K. Furdyna, A. V. Akimov, and M. Bayer, Phys. Rev. B 85, 195324 (2012).

${ }^{7}$ A. Lemaitre, A. Miard, L. Travers, O. Mauguin, L. Largeau, C. Gourdon, V. Jeudy, M. Tran, and J.-M. George, Appl. Phys. Lett. 93, 021123 (2008).

${ }^{8}$ M. Cubukcu, H. J. von Bardeleben, K. Khazen, J. L. Cantin, O. Mauguin, L. Largeau, and A. Lemaitre, Phys. Rev. B 81, 041202 (2010).

${ }^{9}$ A. Werpachowska and T. Dietl, Phys. Rev. B 82, 085204 (2010).

${ }^{10}$ J. Mašek, J. Kudrnovský, F. Máca, J. Sinova, A. MacDonald, R. Campion, B. Gallagher, and T. Jungwirth, Phys. Rev. B 75, 045202 (2007).

${ }^{11}$ J. Xu and M. van Schilfgaarde, J. Magn. Magn. Mater. 305, 63 (2006).

${ }^{12}$ P. R. Stone, K. Alberi, S. K. Z. Tardif, J. W. Beeman, K. M. Yu, W. Walukiewicz, and O. D. Dubon, Phys. Rev. Lett. 101, 087203 (2008).

${ }^{13}$ M. Cubukcu, H. J. von Bardeleben, J. L. Cantin, I. Vickridge, and A. Lemaitre, Thin Solid Films 519, 8212 (2011).

${ }^{14}$ C. Gourdon, A. Dourlat, V. Jeudy, K. Khazen, H. von Bardeleben, L. Thevenard, and A. Lemaître, Phys. Rev. B 76, 241301 (2007).

${ }^{15}$ S. Haghgoo, M. Cubukcu, H. J. von Bardeleben, L. Thevenard, A. Lemaitre, and C. Gourdon, Phys. Rev. B 82, 041301 (2010).

${ }^{16}$ N. Tesarova, D. Butkovičová, R. P. Campion, A. W. Rushforth, K. W. Edmonds, P. Wadley, B. L. Gallagher, E. Schmoranzerova, F. Trojanek, P. Maly, P. Motloch, V. Novak, T. Jungwirth, and P. Nemec, Phys. Rev. B 90, 155203 (2014).

${ }^{17}$ X. Liu, Y. Zhou, and J. Furdyna, Phys. Rev. B 75, 195220 (2007).

${ }^{18}$ D. Wang, Y. Ren, X. Liu, J. Furdyna, M. Grimsditch, and R. Merlin, Phys. Rev. B 75, 233308 (2007).

${ }^{19}$ P. Nĕmec, V. Novák, N. Tesarová, E. Rozkotová, H. Reichlová, D. Butkovičová, F. Trojánek, K. Olejník, P. Malý, R. P. Campion, B. L. Gallagher, J. Sinova, and T. Jungwirth, Nat. Commun. 4, 1422 (2013).

${ }^{20}$ C. Kittel, Phys. Rev. B 110, 1295 (1958).

${ }^{21}$ M. van Kampen, C. Jozsa, J. Kohlhepp, P. LeClair, L. Lagae, W. de Jonge, and B. Koopmans, Phys. Rev. Lett. 88, 227201 (2002).

${ }^{22}$ M. Farle, Rep. Prog. Phys. 61, 755 (1998).

${ }^{23}$ J. Smith and H. G. Beljers, Phillips Res. Rep. 10, 113 (1955).

${ }^{24}$ G. T. Rado and J. R. Weertman, J. Phys. Chem. Sol. 11, 315 (1959).

${ }^{25}$ L. Thevenard, C. Gourdon, S. Haghgoo, J.-P. Adam, H. J. von Bardeleben, A. Lemaitre, W. Schoch, and A. Thiaville, Phys. Rev. B 83, 245211 (2011). 